\title{
Cytocompatible scaffolds of poly(L-lactide)/reduced graphene oxide for tissue engineering
}

\author{
Esperanza Díaz ${ }^{1,2}$, Naroa Iglesias ${ }^{1}$, Sylvie Ribeiro ${ }^{3,4}$, Senentxu Lanceros-Méndez ${ }^{2,5}$ \\ ${ }^{1}$ Escuela de Ingeniería de Bilbao, Departamento de Ingeniería Minera, Metalúrgica y Ciencia \\ de Materiales, Universidad del País Vasco (UPV/EHU) 48920 Portugalete, Spain. \\ ${ }^{2}$ BCMaterials, Basque Centre for Materials, Applications and Nanostructures, UPV/EHU Science \\ Park, 48940 Leioa, Spain \\ ${ }^{3}$ Centro de Física, Universidade do Minho, 4710-057 Braga, Portugal; \\ ${ }^{4}$ Centre of Molecular and Environmental Biology (CBMA), Universidade do Minho, 4710-057 \\ Braga, Portugal \\ ${ }^{5}$ IKERBASQUE, Basque Foundation for Science, 48009 Bilbao, Spain
}

\begin{abstract}
Poly(L-lactide)/reduced Graphene oxide (PLLA/rGO) scaffolds were studied in the present work. The scaffolds were fabricated by TIPS (thermally induced phase separation). Nonisothermal crystallization study for PLLA/rGO was investigated and revealed the nucleating effect of $\mathrm{rGO}$. rGO effect on cytotoxicity, thermal properties, and hydrolysis resistance of PLLA and PLLA/rGO scaffolds were analysed. In vitro degradation in phosphate-buffered solution at $37^{\circ} \mathrm{C}$ is analyzed over twelve weeks. A high crystalline behaviour reduces the speed of hydrolysis and therefore implies less variation in $\mathrm{pH}$, mass loss and water up take. The rGO does not seem to accelerate the degradation process. Finally, rGO contents in PLLA up to $1 \mathrm{wt} \%$ dos not lead to cytotoxic effect, the scaffolds supporting cell adhesion and proliferation.
\end{abstract}

\section{Introduction}

Since their discovery in 2004, graphene family materials (GFFMs) have been widely studied. The exceptional properties of reduced graphene oxide $(\mathrm{rGO})$ and graphene oxide $(\mathrm{GO})$ have led to the development of numerous composites for different biomedical applications, including biosensors (1), drug delivery (2), scaffolds for bone regeneration ( 3 ) or structural applications (4), among others. 
By adding $\mathrm{rGO}$ to polymers some properties are improved such as mechanical, thermal, or electrical properties, among others (5). One of the disadvantages of rGO is its cytotoxicity, which depends on whether it is functionalised and on the functional groups, concentration and also on the manufacturing processes (3). Different scaffold manufacturing techniques have been used, such as 3D printing, supercritical $\mathrm{CO}_{2}$-assisted phase inversión, laser sintering or freeze drying (6).

Recently, the development of artificial bone is being of great interest to researchers, scaffolds of polymer composites with $\mathrm{GO}$ and $\mathrm{rGO}$ are being used for this purpose. Some of the polymers used have been collagen. In the study carried out by Kanayama et al. (7), the bioactivity of collagen/go and collagen/rGO was compared and it was found that scaffolds with rGO are more bioactive than those with $\mathrm{GO}$. Wang et al $(8,9)$ studied the crystallization kinetics and morphology of PLLA/ GO nanocomposites and concluded that nonisothermal and isothermal melt crystallization are influenced by the GO loading and the crystallization mechanism and crystal structure remain unchanged for PLLA and its composites. Other polymers used have been chitosan (10), alginate (11), PVA (polyvinyl alcohol) (12), showing that the addition of up to $1 \%$ GO significantly increases the mechanical properties. Chen et al (13) studied the anisotropy of the properties of polyurethane/poly (lactic acid) when graphene oxide was added using the 3D printing manufacturing technique.

PLLA is a thermoplastic belonging to the family of aliphatic polyesters. It has attracted the attention of researchers because its properties such as: biocompatible, biodegradable (PLLA is degraded by simple hydrolysis of the ester bond), can be synthesized from renewable sources and it is resistant to microbial attack. (17). A drawback of PLLA is the lack of suitable mechanical properties due to its low degree of crystallinity. Thus, nucleating agents ahve been introduced in the scaffolds in order to improve the crystallinity. Those nucleating agents include multiwalled carbon nanotubes, GO and rGO $(8,9)$.

In numerous studies it has been found that the addition of graphene oxide and reduced oxide graphene improve the adhesion, differentiation and proliferation of osteoblasts in scaffolds (3). Cheng et al. (14) found a biomimetic mineralization of hydroxyapatite (HA) during bone formation in PDA (polydopamine)/rGO. This substrate showed improved cellular activity than that of GO. Tapas et al.(15) studied the application of graphene for osteogenic control and differentiation of human mesenchymal stem cells, finding that the presence of graphene did not influence the shape and growth of the cells, which demonstrated its biocompatibility. Crowder 
et al. tested how Graphene's 3D sponges for culturing human mesenchymal stem cells promoted osteogenic differentiation (16). Further, tissue regeneration strategies for muscle and other electrically responsive tissues are also increasingly relying on electrically active microenvironments $(X X)$, being therefore necessary the development of new generation of scaffolds, such and the ones based on $\mathrm{rGO}$, with tailored electrical response.

In the bibliography, it can be found numerous investigations of PLLA/GO, but GO and rGO do not show the same behaviour. The big difference between GO and rGO is the number of oxygen molecules present, influencing both surface interactions and electrical conductivity. GO is an insulator, while $\mathrm{rGO}$ is electrically conductive. The conductivity depends on the degree of reduction (18). There are very few results on the effects of rGO on PLLA ageing and degradation behavior. In this paper, we discussed the effect of addition of reduced oxide Graphene to PLLA scaffolds. Its effect in the citotoxicity and the degradation mechanism was analyzed by discussing morphology, changes in $\mathrm{pH}$, weight loss and thermal properties. This new scaffold with properties like controlled noncytotoxic, biodegradation and biocompatibility are essentials for tissue regeneration.

\section{Experimental Details}

\subsection{Materials}

- Poly(L-lactide) (PLLA) ( $\mathrm{Mw}=142,00, \mathrm{Mn}=95,700$ and $\mathrm{I}=1.5)$ was supplied by Byomer $\mathrm{L} 9000$ (Germany). PLLA was charactericed by gel permeation chromatography (GPC, Perkin Elmer 200) with THF as solvent. Reduced Graphene oxide with an average particle size of 6-7 $\mu \mathrm{m}$ was supplied by Graphenea (San Sebastian, España). 1,4 Dioxane was used as solvent (Panreac p.a., Barcelona, Spain). Phosphate buffer solution (PBS), was supplied by Fluka Analytical (Sigma Aldrich, USA) at a pH of 7.2, was used as the degradation fluid.

\subsection{Scaffolds fabrication and in vitro degradation}

Porous scaffolds were prepared with $2.5 \%(\mathrm{w} / \mathrm{v})$ of PLLA in 1,4-dioxane. The solution was stirred at $50 \stackrel{\circ}{ } \mathrm{C}$ for $2 \mathrm{~h}$ to dissolve the polymer. Then the $\mathrm{rGO}$ was dispersed by ultrasonic stirring. This solution with different $\mathrm{rGO}$ proportions of total polymer mass $(0 \%, 0.3 \%, 0.6 \%, 1 \%)$ was freezedried (LyoQuest of Telstar, Barcelona Spain) for 7 days. The resultant scaffolds are shown in Figure 1. 


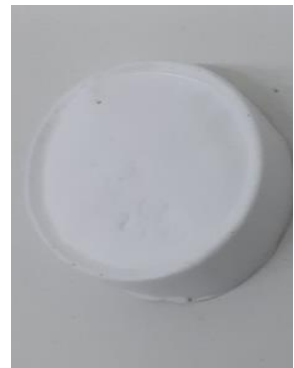

(a)

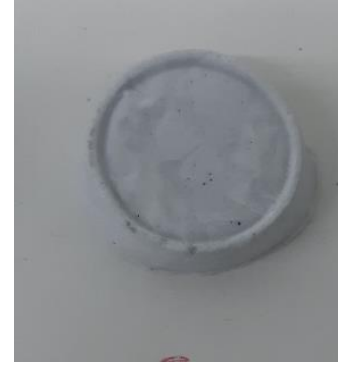

(b)

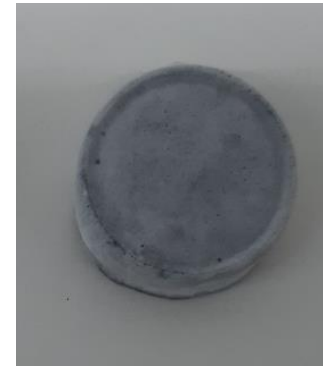

(c)

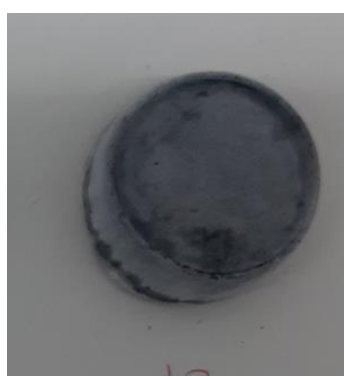

(d)

Figure 1. Macroscopic image of the different types of fabricated scaffolds: (a) PLLA. (b) PLLA/0.3\% rGO. (c) PLLA/0.6\% rGO. (d) PLLA/1\% rGO.

Scaffolds were cut into $0.5 \mathrm{~cm}^{2}$ rectangular samples and measured the initial weight in order to study in vitro degradation in PBS at $37^{\circ} \mathrm{C}$ during 0, 4, 8 and 12 weeks. A PCE $228 \mathrm{pH}$ meter (PCE Instruments, Pons, Alicante, Spain) was used to measure the $\mathrm{pH}$ change in PBS before degradation. The mass loss was calculated according to the following equations:

$$
\begin{aligned}
& \mathrm{W} \mathrm{L} \%=\frac{W o-W r}{W o} \times 100(1) \\
& W a \%=\frac{W w-W r}{W r} \times 100(2)
\end{aligned}
$$

where $W_{L} \%$ is the percentile weight loss and $W a \%$ the water absorption. Wo is the original mass before degradation, $\mathrm{Wr}$ is the weight of a dry simple after degradation and $\mathrm{W} w$ is the weight of the sample before degradation and after removing the surface water.

\subsection{Characterizations}

SEM analysis: Prior to analyze the morphology, the samples were coated with gold, in a JEL Ion Sputter JFC-1100 (Amiron Machinery, Oxnard, CA) at $1200 \mathrm{~V}$ and 5 mA. A Scanning Electron Microscopy (SEM) (HITACHI S-3400N, Tokyo, Japan) was used to study the morphology.

DSC analysis: The thermal properties of the scaffolds were determined with a differential scanning calorimetry (DSC) TA Instruments calorimeter (Waters, NC, USA). Measurements were 
performed at a heating rate of $10 \mathrm{o} \mathrm{C} / \mathrm{min}$ under a nitrogen gas purging system to prevent

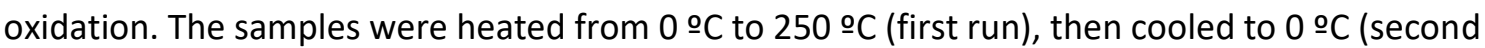
run) and finally heated to $250 \stackrel{\circ}{C}$ (third run). The crystallinity percentage of the samples was calculated with the following equation (19):

$$
\mathrm{Xc} \%=100\left[\left(\Delta \mathrm{Hm}_{1}-\Delta \mathrm{Hc}\right) / \Delta \mathrm{Hm}^{\circ}\right]
$$

Where $\mathrm{Xc}$ is the crystalline fraction (\%), $\Delta \mathrm{Hc}$ the crystallization enthalpy, $\Delta \mathrm{Hm}$ the melting enthalpy and $\Delta \mathrm{H}_{\mathrm{m}}{ }^{\circ}$ is the heat of fusion of $100 \%$ crystalline PLLA (93 J/g) (20).

\subsection{Cytotoxicity tests}

For the in vitro assays, membranes with $0.1 \mathrm{~g} \cdot \mathrm{mL}^{-1}$ were cut and sterilized by $\mathrm{UV}$ for $2 \mathrm{~h}$ before cell seeding ( $1 \mathrm{~h}$ each side). After that, the samples were washed 5 times with PBS solution for 5 min each. The indirect cytotoxicity evaluation of the samples was conducted by adapting the ISO 10993-5 standard test method.

Briefly, the conditioned media were prepared by immersing the samples in 24-well tissue culture polystyrene plate with DMEM containing 4.5 g. $\mathrm{L}^{-1}$ glucose supplemented with $10 \%$ FBS and $1 \%$ $\mathrm{P} / \mathrm{S}$, at $37^{\circ} \mathrm{C}$ in a $95 \%$ humidified air containing $5 \% \mathrm{CO}_{2}$ and incubated for $24 \mathrm{~h}$.

At the same time, the $\mathrm{C} 2 \mathrm{C} 12$ cells were seeded in the 96-well tissue culture polystyrene plate at the density of $2 \times 10^{4}$ cells. $\mathrm{mL}^{-1}$ and incubated for $24 \mathrm{~h}$ to allow the cell attachment on the plate. Then, the culture medium from the 96-well tissue culture polystyrene plate was removed and the conditioned media (from the samples) were added to the wells (100 $\mu \mathrm{L}$ ). Afterward, the cells were incubated for $72 \mathrm{~h}$ and after this time, the evaluation of the cell viability was quantified with(3-(4,5-dimethylthiazol-2-yl)-5-(3-carboxymethoxyphenyl)-2-(4-sulfophenyl)-2H tetrazolium) (MTS) assay. A $20 \%$ of DMSO was used as a positive control and the cell culture medium was employed as negative control.

At the desired time points, the MTS reagent was added into each well in proportion of 1 part to 5 of DMEM medium and, incubated at $37{ }^{\circ} \mathrm{C}$ for $2 \mathrm{~h}$. The absorbance was detected at $490 \mathrm{~nm}$ with a microplate reader (Bioteck).

All quantitative results were obtained from four replicate samples and controls, and were analyzed as the average of viability \pm standard deviation (SD).

The percentage of cell viability was calculated with the equation 4 .

$$
\text { Cell viability }(\%)=\frac{\text { absorbance of sample }}{\text { absorbance of negative control }} \times 100
$$




\section{Results and discussion}

\section{Morphology}

The scaffolds fabricated by TIPS have are characterized by an anisotropic tubular morphology. The orientation of the pores is determined by the progression of solvent crystallization. In the micrographs obtained by SEM (Figure 2 ) it can be observed how by adding $0.3 \%$ rGO the morphology of the scaffolds becomes more compact, with the walls of the pores thickened and a greater order of the structure (Figure 1.d). The addition of 0.6 and $1 \% \mathrm{rGO}$, however, increases the pore size, as shown in Figures $1 \mathrm{~g}$ and $1 \mathrm{j}$ respectively. When rGO particles were added to the polymeric solution, the crystallization of the solvent was modified, the growth process of the crystals changed and a more anisotropic structure and irregular pores were formed (21).

If the pore is larger, the sample should absorb more water and therefore the degradation process should be accelerated. But it should be taken into account that most aliphatic polyesters undergo an autocatalytic degradation process produced by the degradation products themselves. When the pores are larger it is easier for these products to get out of the scaffold and the autocatalytic degradation is slowed down (22). Micrographs $1 \mathrm{f}, 1 \mathrm{j}$ and $1 \mathrm{k}$ show the plasticisation of the scaffolds by the effect of PBS. In micrographs $1 \mathrm{c}, 1 \mathrm{f}, 1 \mathrm{i}$ and $1 \mathrm{k}$, no degradation process of the scaffolds can be observed after 12 weeks of degradation. The presence of $\mathrm{rGO}$ does not seem to accelerate the degradation process unlike reported for PLLA/GO $(8-9,23)$. The difference between $\mathrm{GO}$ and $\mathrm{rGO}$ is the number of oxygen molecules present. Also, rGO has higher surface area as a result of removal of intercalating oxygen groups during the reduction process.

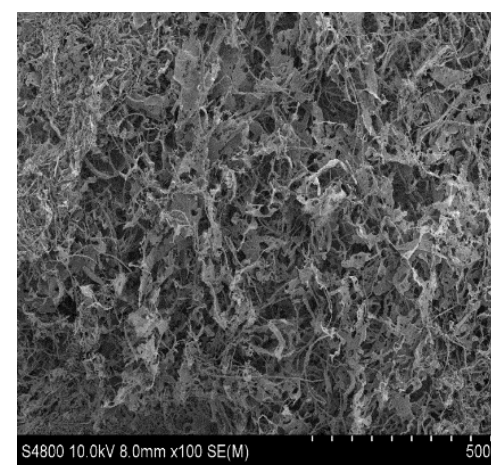

(a)

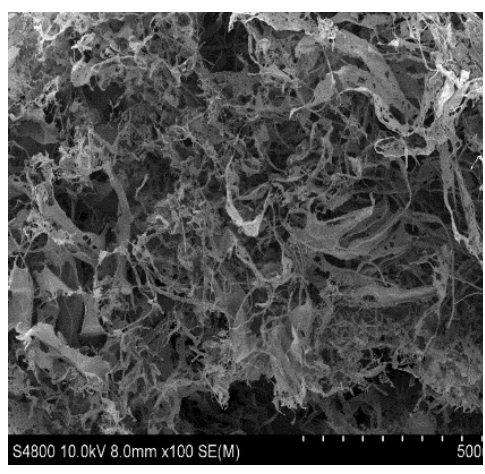

(b)

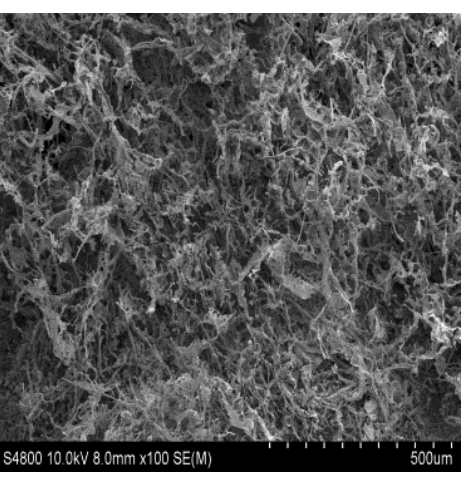

(c) 


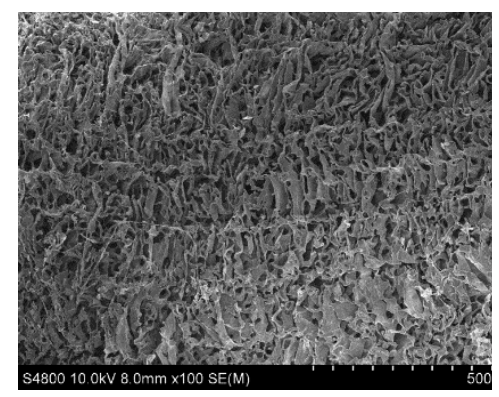

(d)

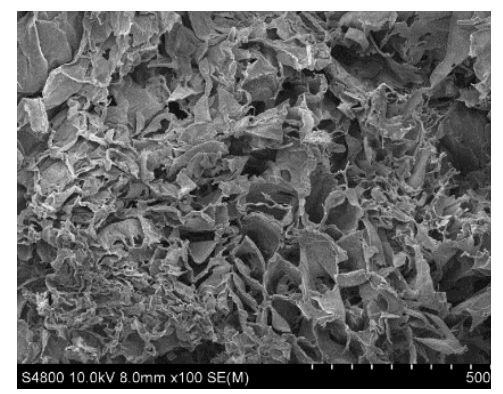

(g)

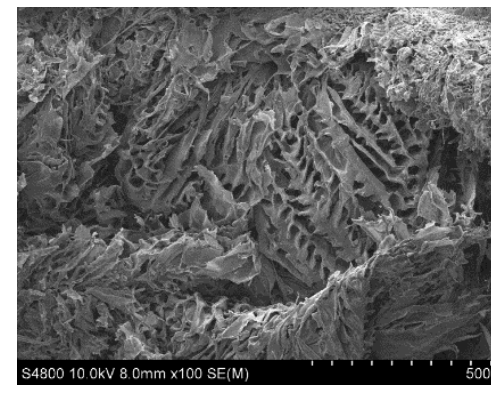

(j)

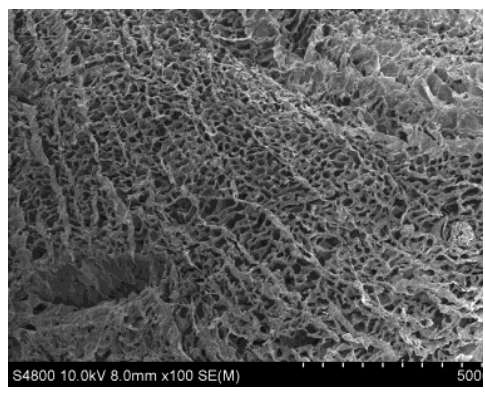

(e)

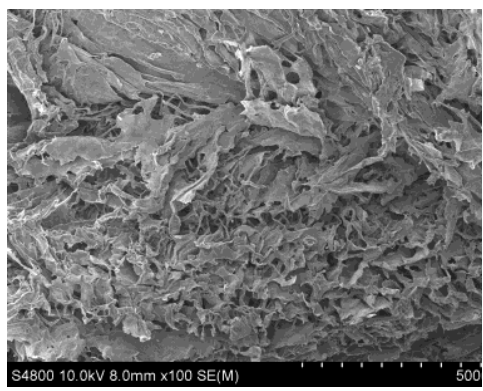

(h)

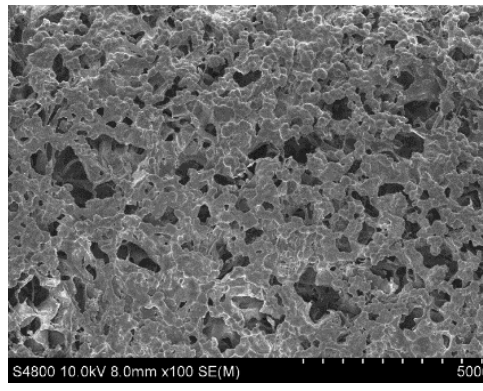

(j)

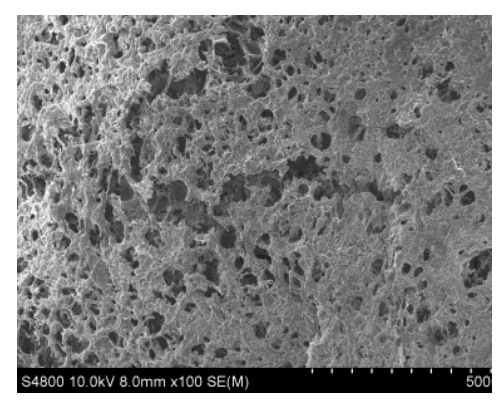

(f)

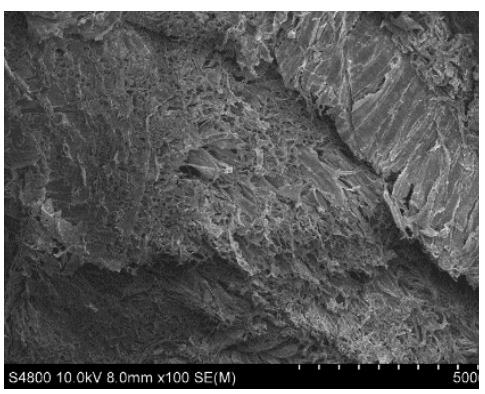

(i)

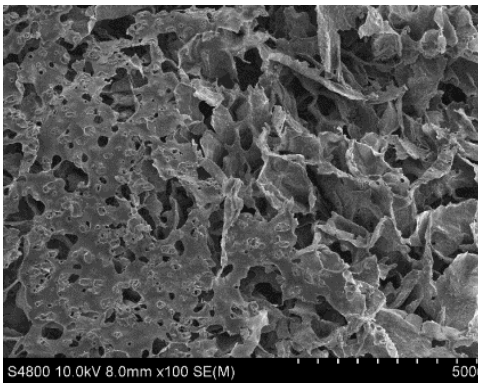

(k)

Figure 2. SEM observation of surface morphology of PLLA. (a) PLLA before degradation. (b) PLLA after in vitro degradation for eight weeks. (c) PLLA after in vitro degradation for twelve weeks. (d) PLLA/rGO 0.3\% before degradation. (e) PLLA/rGO 0.3\% after in vitro degradation for eight weeks. (f) PLLA/rGO 0.3\% after in vitro degradation for twelve weeks. (g) PLLA/rGO $0.6 \%$ before degradation. (h) PLLA/rGO $0.6 \%$ after in vitro degradation for eight weeks.(i) PLLA/rGO $0.6 \%$ after in vitro degradation for twelve weeks. (j) PLLA/rGO 1\% before degradation. (j) PLLA/rGO $1 \%$ after in vitro degradation for eight weeks. ( $k$ ) PLLA/rGO $1 \%$ after in vitro degradation for twelve weeks.

\section{DSC analysis}

Nonisothermal melt crystallization behaviour, glass transition temperatura ( $\mathrm{Tg}$ ) and melting

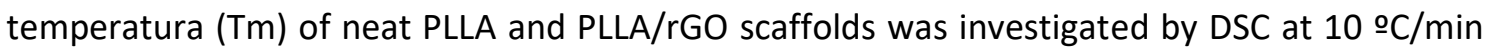


(Figure 3). Table 1 summarizes the thermal parameters before and after in vitro degradation. rGO addition leads to an increase of the glass transition temperatura $\mathrm{Tg}$ in $\approx 5 \stackrel{\circ}{ } \mathrm{C}$. This increase of Tg may be due to the constrained chain mobility of PLLA by hidrogen bonding and electrostatic attraction with rGO (24). Tm, on the other hand, remained unchanged. The cristallization peak temperature (Tc) of neat PLLA is 105 and 129, 135.5 and 136.7 for scaffolds with $0.3,0.6$ and $1 \%$ rGO respectively. The crystallization enthalpy $(\Delta \mathrm{Hc})$ increases from 0.6 for neat PLLA to 31.7, 26.1 and $18 \mathrm{~J} / \mathrm{g}$ for scaffolds with $0.3,0.6$ and $1 \%$ rGO respectively. The crystalline fraction (\%) increases from 12.3 for neat PLLA to 37.9 for $0.3 \mathrm{rGO}$. Tcc, $\Delta \mathrm{Hc}$ and $\% \mathrm{Xc}$ in the PLLA/rGO scaffolds first increases and then decreases with increasing amount of rGO from 0.3 to $1 \mathrm{wt} \%$. The nonisothermal melt crystallization of PLLA has been enhanced significantly in the PLLA/rGo samples relative to neat PLLA. This may indicate that the rGO acts as a nucleating agent for PLLA.

Wang and Qiu have reached the same conclusions in their works $(8,9)$ of nonisothermal and isothermal cristallization of PLLA and PLLA/GO. Also a heating rate of $10 \% / \mathrm{min}$ for both neat PLLA and the PLLA/GO accelerated the nonisothermal cristallization. Valapa et al. (24) studied the nonisothermal cristallization del PLA/graphene and have found similar behaviour. They performed DSC tests at a heating rate of $2.5 \mathrm{o} / \mathrm{min}$ and found a double exotherm melting corresponding to the phenomenon of melting imperfect and perfect crystalline structures of PLA developed in the primary and secondary phase of the cold crystallization process. However, bimodal endotherm melting is not noticed at higher heating rates of $10 \stackrel{\circ}{\circ} / \mathrm{min}$.

After in vitro degradation the samples experience an increase in $\mathrm{Tg}$ with the exception of those with a higher \% rGO (0.6 and 1\%), which decrease. This could be explained as the PLLA is hydrolyzed and shorter chains are formed which can affect its mobility resulting in a lower $\mathrm{Tg}$. However, the PLLA and PLLA / rGO 0.3\% samples show a higher degradation rate and these small chains have crystallized more easily which hinders their mobility and therefore increases $\mathrm{Tg}$. Similar behaviour occurs with Tc. All samples increase their Tc with the crystallization temperature except for PLLA with 0.6 and $1 \%$ rGO, this could be because these samples are in a first stage of the hydrolysis process, which is when products with a shorter chain length are formed and can be more easily packed to form crystals and therefore have a lower crystallization temperature.

As a result of hydrolysis, small chains are formed which crystallise more easily and the Tm and $\Delta \mathrm{Hm}$ should be larger. This would cause less mobility by hydrogen bonding and electrostatic attraction with rGO. This behaviour is experienced by PLLA during the whole degradation period and PLLA/rGO $0.3 \%$ until week 8 of degradation. But the samples with 0.6 and $1 \%$ rGO have a 
lower $\mathrm{Tm}$ and $\Delta \mathrm{Hm}$ as hydrolysis progresses, which would imply a slower degradation rate. Similar results have been observed in $(23,25)$.

\section{$\mathrm{pH}$ variations}

The $\mathrm{pH}$ is an indicator of the acid waste generated with the hydrolytic degradation of the ester groups present, therefore it is important to study the modifications that the $\mathrm{pH}$ undergoes in this process. The inflammatory response of a tissue is highly dependent on the $\mathrm{pH}$ variations of the medium. Figure $4 a$ shows the evolution of this parameter. All samples experience an increase in $\mathrm{pH}$ until the fourth week of degradation, with the highest value being the sample of PLLA and PLLA_0.3\% rGO, and the lowest being for the samples PLLA_1\% rGO and PLLA_0.6\% rGO. They then experiencer a decrease until the eighth week of degradation, to tend to stabilize at the original value in the twelfth week of degradation.

The values obtained seem to indicate that the addition of $\mathrm{rGO}$ tends to stabilize the $\mathrm{pH}$ value, being the samples with higher rGO contents ( 1 and $0.6 \%$ ), the ones that suffer the least variations with respect to the initial value of 7.2. This may be related to the role of reduced graphene oxide as a nucleating agent. A high crystalline behaviour reduces the speed of hydrolysis and therefore implies less variation in $\mathrm{pH}$. A similar result has been found by Girdthep et al (23) in their studies with poly(L-lactic acid)/Graphene nanoplatelets.

\section{PBS uptake}

Figure $4 \mathrm{~b}$ shows the variation of absorbed PBS as a function of degradation time. Up to the fourth week, all samples absorb PBS in large quantities and the absorbed percentage is inversely proportional to the rGO content. From the fourth to the eighth week, they show a tendency to stabilize except for PLLA. From the eighth to the twelfth week the percentage of absorbed PBS increases again except for the sample with the highest rGO content, which has a stable trend. This behaviour coincides with that observed for $\mathrm{pH}$ (figure $\mathrm{X}$ ). Other authors have found an opposite behaviour but for the PLLA/GO system (25). This could be attributed to the fact that GO has many hydroxyl, epoxy, carbonyl and carboxylic acid groups randomly distributed within the GO sheets. These groups make GO very hydrophilic and reactive. By eliminating, in the reduction of GO functional groups, the hydrophobicity of the rGO increases. It should be noted that there might be remaining functional groups in the $\mathrm{GGO}$ resulting from incomplete reduction. 


\section{Mass loss}

In figure $4 c$, it can observed the variation of lost mass as a function of the degradation time. The trend of all samples is practically the same. All samples experience a mass loss of less than $12 \%$ until the fourth week of degradation, being the sample that loses the most mass that of PLLA and from this moment, the rate of degradation slows down to values below $10 \%$.

It can be stated that the rGO seems to slow down the degradation process from the fourth week of degradation. Ma et al. (25) have found in their studies of PLLA/GO composites manufactured by electrospinning that the presence of graphene in the matrix has an accelerating effect on PLLA degradation proportional to GO content, but this effect is just reported for the first 9 days of degradation.

Shuai et al (26) focused their research on the acceleration of degradation due to GO. This, due to its good hydrophilic properties and water absorption capacity, acted as an inducer of ester bonds hydrolysis. That is, with the water present in PBS, hydrogen bonds are formed between the functional groups of GO and the ester bonds of PLLA, causing the cleavage of the macromolecular chains. Our system has $\mathrm{rGO}$, which is a GO reduction product and has a more hydrophobic character. In previous hydrolysis studies carried out by the research group for PLLA / MWCNTs scaffolds (27), a similar behavior was found in the first 12 weeks of degradation.

Table 1. Parameters obtained by thermal analysis on the PLLA/rGO system: $d t$ (weeks) = degradation time in weeks, $\mathrm{Tm}=$ melting point $\left({ }^{\circ} \mathrm{C}\right), \Delta H m=$ melting enthalpy $(\mathrm{kJ} / \mathrm{kg}), \mathrm{Tg}=$ glass transition temperature $\left({ }^{\circ} \mathrm{C}\right), \mathrm{Tc}=$ crystallization temperature $\left({ }^{\circ} \mathrm{C}\right), \Delta \mathrm{Hc}=$ crystallization enthalpy $(\mathrm{kJ} / \mathrm{kg}), X_{c}=$ crystalline fraction (\%), calculated as $\mathrm{Xc} \%=100[(\Delta \mathrm{Hm} 1-\Delta \mathrm{Hcc}) / \Delta \mathrm{HmO}]$ with $\Delta \mathrm{Hm}_{0}$ $=93 \mathrm{~J} / \mathrm{g}[16], C C=$ crystallization capacity (\%), calculated as: $C F \%=100(\Delta \mathrm{Hc} / \Delta \mathrm{Hm} 1)$.

\begin{tabular}{|c|c|c|c|c|c|c|c|}
\hline Samples & $\begin{array}{l}\text { D.Times } \\
\text { (weeks) }\end{array}$ & $\operatorname{Tg}(C)$ & $\operatorname{Tm}(-\mathrm{C})$ & Tc (ㅇ) & $\begin{array}{l}\Delta \mathrm{Hc} \\
(\mathrm{J} / \mathrm{g})\end{array}$ & $\begin{array}{l}\Delta \mathrm{Hm} \\
(\mathrm{J} / \mathrm{g})\end{array}$ & $\mathrm{Xc} \%$ \\
\hline \multirow[t]{4}{*}{ PLLA } & 0 & 60.5 & 168 & 105 & 0.62 & 11.5 & 12 \\
\hline & 4 & 63.5 & 169 & 134 & 18 & 34 & 36.5 \\
\hline & 8 & 65 & 169 & 134 & 20 & 23.5 & 25 \\
\hline & 12 & 63 & 169 & 134 & 21 & 36.5 & 39 \\
\hline PLLA/rGO 0.3\% & 0 & 64.5 & 169.5 & 129 & 32 & 35 & 38 \\
\hline
\end{tabular}




$\begin{array}{cccccccc} & 4 & 65.5 & 172.5 & 131.5 & 29 & 35.5 & 38.5 \\ & 8 & 65.5 & 170.5 & 134 & 30.5 & 35.5 & 38.5 \\ & 12 & 64 & 170.5 & 133 & 20.5 & 26.7 & 29 \\ \text { PLLA/rGO 0.6\% } & 0 & 65 & 168.5 & 135.5 & 26 & 33 & 35.5 \\ & 4 & 61.5 & 169 & 133 & 27 & 35 & 37.4 \\ & 8 & 64 & 169.5 & 136 & 37.7 & 36 & 38.5 \\ & 12 & 64 & 169.3 & 134.5 & 25.5 & 34.5 & 37 \\ & 0 & 66.2 & 168.7 & 137 & 18 & 30.4 & 32.5 \\ & 4 & 65 & 169.7 & 136 & 24.5 & 35.5 & 38.3 \\ & 8 & 64.5 & 169 & 134 & 38 & 35 & 38 \\ & 12 & 64.1 & 169 & 135.5 & 22.3 & 33 & 35\end{array}$

\section{Cytotoxicity}

The main goal of this study is to identify the potential cytotoxicity of the PLLA samples with different concentration of $\mathrm{rGO}$ and, therefore, provide relevant information for further biomedical applications. The cytotoxicity of PLLA and PLLA-rGO samples was evaluated, according the ISO standard 10993-5, with C2C12 cells. Figure 5 demonstrated the results obtained after $72 \mathrm{~h}$.

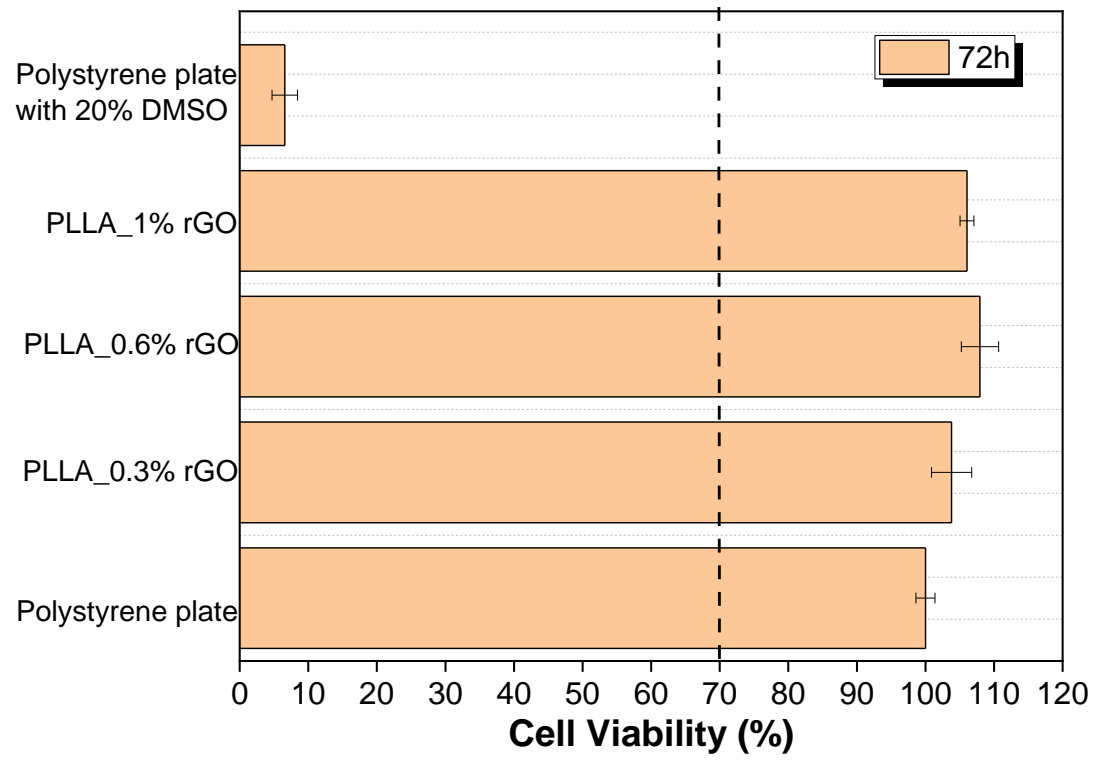


Figure 5. Cell viability of $\mathrm{C} 2 \mathrm{C} 12$ cells in contact with the conditioned media exposed with different samples up to $72 h$.

For tissue engineering and biomedical applications, it is essential to proceed to the evaluation of the material cytotoxicity. PLLA is a biocompatible and biodegradable polymer, being one of the most used polymers for biomedical applications in the human body (28). The introduction of $\mathrm{rGO}$ particles on the polymer will allow the development of new platforms for biomedical applications. It is demonstrated in the literature that the cytotoxicity of the graphene oxide depends of the used processing techniques (29). Analyzing the figure 5 , it is observed that after $72 \mathrm{~h}$, the composites with different rGO content up to $1 \%$ in the PLLA matrix are not cytotoxic for $\mathrm{C} 2 \mathrm{C} 12$ cells, so in this sense, they can be used for cell proliferation assessment in direct contact in further experiments. According to the ISO standard 109935-5, samples are considered cytotoxic when the cell viability, as compared to the control, present values below $70 \%$. It is reported in the literature that $\mathrm{GO}$ concentrations between 25 and $50 \mu \mathrm{g}-\mathrm{ml}^{-1}$ increase the viability, metabolic activity and membrane integrity of encapsulated C2C12-erythropoietin myoblasts (30). Thus, it has been demonstrated that the introduction of 1 wt\% rGO in PLLA fibre matrix did not exhibit cytotoxicity supporting cell adhesion and proliferation (31), what confirm the obtained results. These results confirm the suitability of the composites for advanced tissue regeneration strategies (32).

\section{Acknowledgements}

Work supported by the Portuguese Foundation for Science and Technology (FCT): projects UID/FIS/04650/2020, PTDC/EMD-EMD/28159/2017 and PTDC/BTM-MAT/28237/2017. Financial support from the Spanish State Research Agency (AEI) and the European Regional Development Fund (ERFD) through the project PID2019-106099RBC43/AEI/10.13039/501100011033 and from the Basque Government Industry and Education Departments under the ELKARTEK and PIBA (PIBA-2018-06) programs, respectively, are also acknowledged. 